\section{Subnotificação da mortalidade materna no Estado do Paraná, Brasil: 1991-2005}

\author{
Underreporting of maternal deaths in Paraná State, \\ Brazil: 1991-2005
}

1 Secretaria de Estado da Saúde do Paraná, Curitiba, Brasil.

2 Departamento de Enfermagem, Universidade Tuiuti do Paraná, Curitiba, Brasil.

3 Departamento de

Ginecologia e Obstetrícia,

Universidade Estadual de

Londrina, Londrina, Brasil.

Correspondência V. M. N. Soares

Secretaria de Estado da Saúde do Paraná.

Rua Plácido Prevedello 266, Curitiba, $P R$

82200-420, Brasil.

vaniam@onda.com.br

\section{Abstract}

The objective of this article was to analyze the underreporting of maternal deaths in Paraná State, Brazil, from 1991 to 2005, identifying details on the basic causes that keep maternal deaths hidden and problems with completing items 43 and 44 on death certificates during $a$ recent period (2003-2005). Data were obtained from the database of the Paraná State Committees for the Prevention of Maternal Mortality, which investigate maternal deaths using the Reproductive Age Mortality Survey (RAMOS). According to the data, maternal mortality decreased by $26 \%$ in 15 years, but the level of underreporting remained the same, generating adjustment factors ranging from 1.6 to 1.8. In conclusion, medical schools and the State medical board should provide more incisive and comprehensive orientation on completing death certificates in order to minimize this underreporting. The Paraná State Committees for the Prevention of Maternal Mortality help correct underreporting of maternal deaths by identifying their real causes and determinants and defining strategies to reduce them. More effective measures to reduce avoidable maternal deaths are still needed in the State.

Maternal Mortality; Underregistration; Death Certificates
Vânia Muniz Néquer Soares 1,2

Elbens M. Minorelli de Azevedo 3

Terezinha L. Watanabe 1

\section{Introdução}

A subenumeração dos óbitos maternos prejudica a formulação de medidas adequadas à saúde materna $1,2,3$.

Morte materna segundo a Organização Mundial da Saúde (OMS) é aquela que ocorre durante a gestação ou dentro de um período de 42 dias após o término da mesma, independente da duração ou da localização da gravidez, em função de qualquer causa relacionada (obstétricas diretas) ou agravada pela gravidez (obstétricas indiretas), ou por medidas tomadas referentemente a ela, porém não devidas a causas acidentais ou incidentais (não obstétricas) 4 .

As dificuldades relativas à obtenção de informações sobre essas mortes, quanto ao número e reais causas das mortes, estão diretamente ligadas às incorreções técnicas no preenchimento das Declarações de Óbito 5,6,7. Países desenvolvidos, que contam com bons sistemas de registros, também apresentam erros na atribuição da causa de morte materna nas Declarações de Óbito, dificultando a sua mensuração 3,7,8.

Na França, em 2000, a taxa oficial de mortalidade materna de 8/100 mil nascidos vivos, foi corrigida pelo fator de ajuste de 2,0 identificado em estudo nacional, ficando estimada em 17/ 100 mil nascidos vivos; no Canadá, no mesmo ano, a taxa oficial de 4/100 mil nascidos vivos corrigida por um fator de ajuste de 1,5 passou para 6/100 mil nascidos vivos 3,7 . 
A freqüente subnotificação de óbitos maternos é atribuída ao desconhecimento das regras de preenchimento da Declaração de Óbito e de sua importância nas estatísticas vitais, e também ao ocultamento da causa real das mortes maternas por possibilidade de associação à negligência, imperícia ou a possível ato ilegal 7 .

Dentre as estratégias sugeridas pela OMS e pelo Ministério da Saúde, para correção da subnotificação de tais óbitos, está a criação de Comitês de Prevenção da Mortalidade Materna, que têm como uma de suas atribuições realizar a vigilância dos óbitos maternos, mediante inquéritos ou investigações sobre tais mortes, visando determinar a real magnitude deste agravo e propor medidas para sua redução 1,9.

A OMS define ainda inquérito da mortalidade materna como a investigação anônima, de caráter multidisciplinar, realizada de forma sistemática e com base em provas representativas sobre as condições em que ocorrem as mortes maternas numa determinada área, estado ou país, com o objetivo de identificar o número, causas e fatores evitáveis ou remediáveis associados a estes óbitos 2 .

No Brasil, o Ministério da Saúde desde 1987 vem incentivando a implantação de comitês nos estados e municípios, para que realizem o monitoramento da mortalidade materna e o acompanhamento e avaliação das políticas de assistência à saúde da mulher 9,10 .

O primeiro Comitê Nacional de Mortalidade Materna foi oficializado no Brasil, em 1994, pela Portaria $n^{\circ}$. 773/MS; e em 1997 o óbito materno passou a ser considerado um evento de notificação compulsória em todo território nacional, conforme a Resolução no ${ }^{\circ}$. 256/MS 9,10. No entanto, a notificação não vem ocorrendo de forma sistemática, uma vez que não foram instituídos os mecanismos para facilitar e agilizar a notificação pelos serviços.

O Brasil tem apresentado grandes avanços nos registros oficiais de óbitos, no Sistema de Informação sobre Mortalidade (SIM) do Ministério da Saúde, atingindo uma cobertura de 90,4\% dos óbitos, em 2004. A cobertura, porém, não é homogênea, as regiões Norte e Nordeste do país têm registrado apenas cerca de $70 \%$ dos seus óbitos 11 .

Por conta dessas diferenças, o Ministério da Saúde, a partir de 2000, passou a incluir para o cálculo da Razão de Mortalidade Materna (RMM) para o Brasil, apenas os dados dos estados com cobertura do SIM, igual ou superior a $90 \%$ (os estados das regiões Sudeste, Sul - que inclui o Paraná - e do Centro-Oeste, com exceção do Mato Grosso), chegando a uma RMM de 76,09 por 100 mil nascidos vivos, em 200411.
A OMS, utilizando outras metodologias estatísticas, estima que as taxas brasileiras sejam muito superiores, ficando entre 126 a 260 mortes por 100 mil nascidos vivos; contudo essas estimativas são questionadas por pesquisadores e autoridades brasileiras 3,5 .

O Paraná possui um bom sistema de informação de mortalidade e de nascimentos e realiza a correção da subnotificação da mortalidade materna desde 1989, utilizando as informações dos Comitês Municipais e Regionais de Prevenção da Mortalidade Materna, que são assessorados pelo Comitê Estadual de Prevenção da Mortalidade Materna. Valendo-se da metodologia Reproductive Age Mortality Study (RAMOS), os comitês investigam a cada ano, aproximadamente $90 \%$ dos 3 mil óbitos de mulheres em idade fértil (10-49 anos) que ocorrem no estado, identificando os óbitos maternos por meio de pesquisa em múltiplas fontes, dentre elas prontuários ambulatoriais, hospitalares, laudos de necropsia e entrevistas com familiares e profissionais envolvidos. Após investigação e confirmação de cada óbito materno nas Câmaras Técnicas dos Comitês para Estudo dos Óbitos Maternos, eles são incluídos no SIM, corrigindo os dados oficiais 12 .

A redução da mortalidade materna tem sido uma preocupação mundial, e com esta perspectiva a Organização das Nações Unidas (ONU) estabeleceu em 2000 como um dos oito Objetivos de Desenvolvimento deste Milênio, a redução em $75 \%$ da RMM até 2015, tendo como referência a RMM de 199013

Constitui-se por outro lado um desafio tanto quantificar a redução da mortalidade materna nos diferentes países e regiões por causa da subnotificação dos óbitos, quanto definir os investimentos e ações para a redução destas mortes, já que os seus determinantes precisam ser identificados, indo além de sua simples quantificação 2 .

Em 2005 uma primeira avaliação dos progressos na consecução dos Objetivos de Desenvolvimento deste Milênio ressalta que a mortalidade materna permanece inaceitavelmente alta em todas as regiões em desenvolvimento, refletindo a falta de atenção pública às necessidades das mulheres e acesso inadequado a informações e serviços de saúde sexual e reprodutiva, incluindo serviços obstétricos de emergência. A América Latina, segundo tal avaliação, vem apresentando progressos muito lentos em relação ao objetivo proposto 13 .

O Governo Brasileiro, na qualidade de Estado-Parte das Nações Unidas, tem assumido compromissos na elaboração e execução de políticas públicas que promovam a maternidade segura $\mathrm{e}$ garantam os direitos sexuais e reprodutivos. 
Objetivando cumprir os compromissos, o Ministério da Saúde lançou em 2004 o Pacto Nacional para Redução da Mortalidade Materna e Neonatal, que propõe a execução de um conjunto de ações articuladas das diferentes esferas de governo, para a qualificação da atenção obstétrica e neonatal. O pacto propõe, para isso, a melhoria dos registros dos óbitos maternos para conhecer sua real magnitude e o desenvolvimento de ações para enfrentar as causas passíveis de resolubilidade nos serviços de saúde 14 .

O sucesso do pacto depende da ampla adesão dos estados e municípios, e da ampla participação dos profissionais.

O presente estudo tem por objetivos analisar a evolução das taxas de subnotificação e do fator de ajuste da mortalidade materna no Estado do Paraná no período de 1991-2005, bem como identificar as causas básicas que ocultam os óbitos maternos e as causas refeitas que foram acrescentadas às estatísticas oficiais em período mais recente, triênio 2003-2005. Complementarmente, para este mesmo período, analisou-se o preenchimento dos campos 43 e 44 da Declaração de Óbito, campos específicos para assinalar se os óbitos eram maternos.

\section{Metodologia}

Foram analisados 2.077 óbitos maternos, ocorridos no período de 1991 a 2005, identificados pelos Comitês de Prevenção à Mortalidade Materna do Paraná, após investigação que usa o método RAMOS.

Não foram incluídos neste estudo os óbitos maternos tardios, ou seja, aqueles que ocorreram em período de 42 dias até um ano após o parto, mais freqüentemente subnotificados, merecendo por suas características um estudo à parte.

Os dados do estudo foram obtidos das Fichas de Investigação Confidencial de Óbitos Maternos, utilizadas pelos comitês há 19 anos, as quais são digitadas em um banco de dados próprio, e dados dos "estudos de caso" que são elaborados pelos comitês. Os estudos de caso relatam a evolução de cada óbito individualmente, com base nos documentos levantados nos inquéritos dos óbitos.

Nos estudos de caso constam informações sobre as condições sócio-econômicas das mulheres, antecedentes obstétricos, dados do prénatal, parto, puerpério, da assistência hospitalar, a causa do óbito na Declaração de Óbito, e a entrevista domiciliar. Esses estudos de caso são analisados posteriormente, em Câmaras Técnicas multiprofissionais e interinstitucionais, que buscam identificar a real causa da morte, seus determinantes, a evitação e medidas de prevenção.

Tais estudos são sigilosos quanto ao nome da paciente e do serviço onde ocorreu o óbito, e são amplamente divulgados no estado, sendo uma das estratégias educativas/informativas dos profissionais utilizadas pelo Comitê Estadual, embora sejam dados de domínio público.

A utilização dos dados do banco de dados e dos estudos de caso para esta pesquisa foi comunicada e autorizada em reunião do Comitê Estadual de Prevenção da Mortalidade Materna, sendo dados secundários e sigilosos.

Calculou-se para este estudo a RMM antes e após a correção da subnotificação dos óbitos maternos, o percentual de sub-registro e a taxa de ajuste para o período de 1991 a 2005, subdividido em cinco triênios.

Considerou-se como morte materna declarada, as declarações de óbito em que constava uma causa específica do capítulo XV (Complicações da Gravidez, Parto ou Puerpério) da Classificação Internacional de Doenças, 10a revisão (CID10), ou quando havia alguma menção ao estado gravídico-puerperal, por ocasião do óbito, escrita por extenso na parte I ou II do atestado médico do óbito, independentemente de estarem preenchidos os campos 43 e 44 da Declaração de Óbito, que foram computados separadamente.

A morte materna foi considerada não declarada quando as informações registradas na Declaração de Óbito não permitiram classificar o óbito como materno, e apenas após investigação é que se descobriu tratar-se efetivamente de um óbito materno, conceito estabelecido no Manual dos Comitês de Prevenção da Mortalidade Materna 9.

As Declarações de Óbito em que apenas o campo 43 (para assinalar se a morte ocorreu durante a gravidez parto ou aborto) e/ou o campo 44 (para assinalar se a morte ocorreu durante o puerpério) estavam preenchidos, sem menção a uma causa materna por extenso no atestado médico do óbito, foram consideradas mortes maternas presumíveis, uma vez que elas só foram confirmadas após investigação.

Para identificar as causas que ocultam as mortes maternas e a qualidade do preenchimento dos campos 43 e 44 da Declaração de Óbito, analisaram-se os óbitos maternos do último triênio, 2003-2005, por ser de um período mais recente e com um número relativamente alto de casos (306 óbitos maternos) que permitiria análises atualizadas e consistentes sobre as causas que mascaram as mortes maternas, e as causas maternas que foram mais acrescentadas às estatísticas oficiais após investigação dos óbitos pelos comitês. 


\section{Resultados}

Ocorreram 2.710.351 nascimentos e 2.077 óbitos maternos, no período de 1991 a 2005, determinando uma RMM de 76,63 por 100 mil nascidos vivos. Do total de óbitos, 857 não estavam declarados no atestado médico de óbito, sendo confirmados apenas após investigação pelos Comitês de Prevenção da Mortalidade Materna no Paraná, correspondendo a uma taxa de subnotificação de $41,26 \%$ e a um fator de ajuste de 1,7 nos 15 anos (Tabela 1).

Analisando-se a evolução dessas taxas em cinco triênios, verificou-se que não houve redução importante da subnotificação, ficando as taxas entre $35,5 \%$ e $45,3 \%$ dos óbitos maternos, gerando fatores de ajuste que oscilaram entre 1,6 e 1,8, segundo os critérios definidos neste estudo (Tabela 1).

Ressalte-se que este fator de ajuste não deve ser aplicado sobre dados oficiais do Paraná porque a Secretaria Estadual de Saúde inclui sistematicamente no SIM os óbitos maternos identificados pelos comitês.

Por outro lado observou-se que a RMM, mesmo após correção dos dados oficiais, apresentou redução de $25,6 \%$, passando de 86,41 por $100 \mathrm{mil}$ nascidos vivos no primeiro triênio para 64,26 por 100 mil nascidos vivos no último triênio, uma lenta e gradual redução (Tabela 1).

No triênio 2003-2005, ocorreram 306 óbitos maternos no Paraná, sendo 171 declarados e 135 não declarados.

Analisando-se detalhadamente os 135 óbitos maternos não declarados ocorridos no último triênio (2003-2005), verificou-se que as causas básicas que mais freqüentemente ocultavam as mortes maternas foram as do grupo de doenças do aparelho circulatório (constavam nas declarações de óbito originais as seguintes causas: car- diopatia, hipertensão arterial, acidente vascular cerebral); e as do grupo de doenças do aparelho respiratório (em que constavam broncopneumonias, embolias, pneumonias), que no conjunto ocultavam $40 \%$ das mortes maternas. O grupo de causas relacionadas ao aparelho digestivo (em que estava declarada apenas apendicite, hepatite, insuficiência hepática), ocultava outros 11,9\% dos óbitos (Tabela 2).

As "causas indeterminadas" (quando se lia na Declaração de Óbito - causa desconhecida, morte sem assistência médica ou na dependência de exames complementares), que indiretamente refletem a qualidade da assistência prestada às mulheres, constavam em 28 Declarações de Óbito (20,8\% dos casos) (Tabela 2). Constatou-se ainda que para apenas metade destes óbitos foi realizada a necropsia, mas que apenas confirmavam que a mulher estava ou esteve grávida, mas não elucidavam muitas vezes a causa básica que levou à morte materna, permanecendo como causa materna de causa indeterminada (Tabelas 2 e 3).

As causas obstétricas diretas tiveram maior índice de subnotificação, conforme demonstrado na Tabela 3 , com 53,3\% dos casos, e as causas indiretas representaram $38,5 \%$ de subnotificação neste triênio. Onze dos 135 casos de mortes maternas não declaradas $(8,1 \%)$ permaneceram como causas indeterminadas em razão da falta de dados nos prontuários e/ou a falhas na assistência das mulheres, não permitindo esclarecer seus diagnósticos.

Dentre as causas obstétricas diretas, os óbitos por conta de hipertensão específica na gestação (pré-eclâmpsia, eclâmpsia) tiveram maior acréscimo com 13 casos, seguidas pelas embolias obstétricas com 12 casos e infecção puerperal com 11 casos. Foi significativa também a subnotificação dos casos de infecções urinárias, hemorra-

Tabela 1

Número de nascidos vivos, óbitos maternos declarados e não declarados na Declaração de Óbito, razão de mortalidade de materna (RMM), percentual de óbitos maternos subnotificados, fator de correção por triênio e total. Paraná, Brasil, 1991-2005.

\begin{tabular}{|c|c|c|c|c|c|c|}
\hline Indicadores & 1991-1993 & 1994-1996 & 1997-1999 & $2000-2002$ & 2003-2005 & Total \\
\hline Número de nascidos vivos & 592.517 & 567.333 & 563.444 & 510.874 & 476.183 & 2.710 .351 \\
\hline Número total de óbitos maternos & 512 & 496 & 440 & 323 & 306 & 2.077 \\
\hline Número de óbitos declarados na Declaração de Óbito & 280 & 320 & 254 & 195 & 171 & 1.220 \\
\hline Número de óbitos maternos não declarados na declaração de óbito & 232 & 176 & 186 & 128 & 135 & 857 \\
\hline RMM por 100 mil nascidos vivos pela declaração de óbito original & 47,26 & 56,40 & 45,08 & 38,17 & 35,91 & 45,01 \\
\hline RMM por 100 mil nascidos vivos após correção da subnotificação & 86,41 & 87,43 & 78,09 & 63,22 & 64,26 & 76,63 \\
\hline Percentual de subnotificação & 45,31 & 35,48 & 42,27 & 39,63 & 44,12 & 41,26 \\
\hline Fator de correção & 1,8 & 1,6 & 1,7 & 1,7 & 1,8 & 1,7 \\
\hline
\end{tabular}


Causas originais nas Declarações de Óbito que ocultavam o óbito materno. Paraná, Brasil, 2003-2005.

\begin{tabular}{|c|c|c|}
\hline Causas que ocultavam as mortes maternas & Total & $\%$ \\
\hline $\begin{array}{l}\text { Doenças do aparelho circulatório (cardiopatias, infarto agudo do miocárdio, insuficiência cardíaca, aneurisma, } \\
\text { hemorragia cerebral, hipertensão arterial, acidente vascular cerebral) }\end{array}$ & 27 & 20,0 \\
\hline $\begin{array}{l}\text { Doenças do aparelho respiratório (broncopneumonia, DPOC, asma, insuficiência respiratória, pneumonia, tuberculose, } \\
\text { embolia e tromboembolia pulmonar) }\end{array}$ & 27 & 20,0 \\
\hline $\begin{array}{l}\text { Doenças do aparelho digestivo (apendicite, pancreatite, trombose mesentérica, cirrose hepática, } \\
\text { insuficiência hepática, hepatite, hemorragia digestiva) }\end{array}$ & 16 & 11,9 \\
\hline Indeterminada (sem assistência médica, causa desconhecida) & 14 & 10,4 \\
\hline Indeterminada (no aguardo de exames complementares) & 14 & 10,4 \\
\hline Doenças do sistema nervoso (crise convulsiva, epilepsia) & 7 & 5,2 \\
\hline Doenças do aparelho urinário (abscesso renal, pielonefrite aguda, insuficiência.renal aguda, infecção urinária) & 6 & 4,4 \\
\hline Septicemia & 6 & 4,4 \\
\hline Endometrite & 4 & 3,0 \\
\hline Parada cardiorrespiratória & 4 & 3,0 \\
\hline Neoplasias (ovário, mama, crânio) & 3 & 2,2 \\
\hline Desnutrição severa & 1 & 0,7 \\
\hline CIVD & 1 & 0,7 \\
\hline Colagenopatia a esclarecer & 1 & 0,7 \\
\hline Diabete mellitus & 1 & 0,7 \\
\hline Falência de múltiplos órgãos & 1 & 0,7 \\
\hline HIV & 1 & 0,7 \\
\hline Púrpura trombocitopênica & 1 & 0,7 \\
\hline Total & 135 & 100,0 \\
\hline
\end{tabular}

gias e complicações anestésicas na gravidez, com nove casos cada.

As causas básicas identificadas nas declarações de óbito, neste triênio, que ocultavam (mascaravam) as mortes maternas por hipertensão na gestação, na declaração de óbito original, foram: embolia, AVC, crise convulsiva, congestão passiva do fígado, tromboembolismo. As que ocultavam as embolias obstétricas foram as cardiopatias, infarto agudo do miocárdio, asma e tromboembolismo pulmonar; e as que ocultavam as infecções puerperais, foram a endometrite, septicemia, broncopneumonia, abscesso uterino, embolia, trombose mesentérica (dados não tabulados).

Dentre as causas obstétricas indiretas, as doenças do aparelho circulatório tiveram um aumento em 15 óbitos (11,1\% dos casos) e as doenças do aparelho respiratório em 10 óbitos $(7,4 \%)$, após investigação adequada, análise e correção da causa básica pelos comitês (Tabela 3).

A Tabela 4 demonstra que os campos 43 e 44 da declaração de óbito, campos específicos para o médico assinalar/informar se a mulher estava grávida, se houve aborto ou se a mesma estava no puerpério nos 12 meses que precederam o óbi- to, estavam preenchidos em $89,2 \%$ dos 306 óbitos maternos do último triênio. No entanto, em $30,7 \%$ das declarações, os campos foram preenchidos de forma incorreta, em relação ao período da gravidez em que ocorreu o óbito e em alguns casos descartava o óbito materno, ao assinalar "não" nos dois campos, quando na verdade tratava-se de óbito materno.

Identificou-se ainda pelos dados do SIM que em mais 53 Declarações de Óbitos os campos 43 e 44 estavam preenchidos, mas estes casos foram descartados como óbito materno, após investigação.

\section{Discussão}

O índice de mortalidade materna é um importante indicador de saúde e seus elevados índices evidenciam distorções das políticas sociais e da atenção à saúde reprodutiva, podendo representar uma violação dos direitos humanos das mulheres 5 .

A RMM no Paraná tem apresentado redução; destarte este decréscimo, sabe-se que muito há a ser feito para alcançarmos níveis próximos aos 
Causas dos óbitos maternos declarados na Declaração de Óbito original e as acrescentadas pelas declarações de óbito refeitas após a investigação. Paraná, Brasil, 2003-2005.

\begin{tabular}{|c|c|c|c|c|c|c|}
\hline \multirow[t]{2}{*}{ Causas } & \multicolumn{2}{|c|}{$\begin{array}{c}\text { Óbitos maternos } \\
\text { declarados na Declaração } \\
\text { de Óbito original }\end{array}$} & \multicolumn{2}{|c|}{$\begin{array}{c}\text { Óbitos maternos } \\
\text { incluídos depois de refeita } \\
\text { a Declaração de Óbito }\end{array}$} & \multicolumn{2}{|c|}{ Total } \\
\hline & $\mathbf{n}$ & $\%$ & $\mathbf{n}$ & $\%$ & $\mathbf{n}$ & $\%$ \\
\hline \multicolumn{7}{|l|}{ Obstétricas diretas } \\
\hline Pré-eclâmpsia, eclâmpsia, HAC sobreposta (O11, O14, O15) & 43 & 25,0 & 13 & 9,6 & 56 & 18,2 \\
\hline Embolia obstétrica (O88) & 15 & 8,8 & 12 & 8,9 & 27 & 8,8 \\
\hline Infecção puerperal (O85) & 14 & 8,2 & 11 & 8,1 & 25 & 8,1 \\
\hline Infecção urinária na gravidez (O23) & 6 & 3,5 & 9 & 6,7 & 15 & 4,9 \\
\hline Hemorragias ante e pós-parto $(\mathrm{O} 45, \mathrm{O} 72)$ & 32 & 18,7 & 9 & 6,7 & 41 & 13,3 \\
\hline Complicação anestésica na gravidez (O74) & 5 & 2,9 & 9 & 6,7 & 14 & 4,6 \\
\hline Transtornos do fígado na gravidez $(\mathrm{O} 26)$ & 1 & 0,6 & 4 & 3 & 5 & 1,6 \\
\hline Aborto (O03) & 15 & 8,8 & 2 & 1,5 & 17 & 5,5 \\
\hline Corioamnionite (O41) & 5 & 2,9 & 2 & 1,5 & 7 & 2,3 \\
\hline Miocardiopatia no puerpério (О90.3) & 0 & 0,0 & 1 & 0,7 & 1 & 0,3 \\
\hline Subtotal & 136 & 79,5 & 72 & 53,3 & 208 & 67,7 \\
\hline \multicolumn{7}{|l|}{ Obstétricas indiretas } \\
\hline Doenças do aparelho circulatório (O99.4) & 13 & 7,6 & 15 & 11,1 & 28 & 9,1 \\
\hline Doenças do aparelho respiratório (O99.5) & 4 & 2,3 & 10 & 7,4 & 14 & 4,6 \\
\hline Doenças do SNC (O99.3) & 0 & 0,0 & 7 & 5,2 & 7 & 2,3 \\
\hline Doenças do aparelho digestivo (O99.6) & 2 & 1,2 & 8 & 5,9 & 10 & 3,2 \\
\hline Outras doenças e afecções específicas. (O99.8) & 3 & 1,8 & 6 & 4,4 & 9 & 2,9 \\
\hline Doenças endócrinas, nutricionais e metabólicas (O99.2) & 0 & 0,0 & 1 & 0,7 & 1 & 0,3 \\
\hline Doença infecciosa complicando a gravidez (O98) & 5 & 2,9 & 2 & 1,5 & 7 & 2,3 \\
\hline Outras doenças do sangue e órgãos hematopoéticos (O99) & 0 & 0,0 & 2 & 1,5 & 2 & 0,6 \\
\hline Outras indiretas & 0 & 0,0 & 1 & 0,7 & 1 & 0,3 \\
\hline Subtotal & 27 & 15,8 & 52 & 38,5 & 79 & 25,7 \\
\hline Morte materna de causa não especificada & 8 & 4,7 & 11 & 8,1 & 19 & 6,5 \\
\hline Total & 171 & 100,0 & 135 & 100,0 & 306 & 100,0 \\
\hline
\end{tabular}

20 por 100 mil nascidos vivos considerados como relativamente baixos pela OMS, ou para nos igualarmos aos países desenvolvidos, que apresentam índices inferiores a 10 óbitos maternos por 100 mil nascidos vivos.

A qualidade e confiabilidade das Declarações de Óbitos vêm sendo examinadas e questionadas em diversos estudos em diferentes países e no Brasil, salientando-se a precariedade no preenchimento das Declarações de Óbito, especialmente das causas relacionadas às complicações da gravidez, parto e puerpério 6 .

Siqueira et al. 6 em estudo realizado na Região Sul do Município de São Paulo, consideraram insatisfatória a qualidade da certificação médica dos óbitos das mulheres em idade fértil, após revisão das causas básicas dos óbitos. $\mathrm{O}$ estudo verificou que havia concordância após revisão dos prontuários e outros documentos em apenas $44,9 \%$ entre os atestados originais e os refeitos. Em virtude dessa revisão, foram acrescentados 22 óbitos maternos aos 23 óbitos originais.

Laurenti et al. 5, em recente estudo realizado nas capitais brasileiras com objetivo de conhecer a qualidade da informação da mortalidade de mulheres em idade fértil e estimar a RMM e fator de ajuste para os dados oficiais brasileiros, analisaram 7.332 óbitos de mulheres de 10 a 49 anos, ocorridos no primeiro semestre de 2000, identificando 239 óbitos maternos. Destes, apenas 144 eram declarados, determinando um fator de ajuste de 1,4 e uma RMM de 54,3 por 100 mil nascidos vivos, para o conjunto das capitais. $\mathrm{O}$ fator de ajuste variou entre as regiões, ficando em 1,08 na Região Norte a 1,83 na Região Sul 5.

O fator de ajuste do Paraná de 1,7 nos 15 anos, e 1,8 no último triênio, estão próximos ao encontrado por Laurenti et al. 5 para a Região Sul. 
Preenchimento dos campos 43 e 44 da Declaração de Óbito. Paraná, Brasil, 2003 a 2005.

\begin{tabular}{|c|c|c|c|c|c|c|c|c|}
\hline \multirow[t]{3}{*}{ Ano } & \multicolumn{6}{|c|}{ Preenchimento dos campos 43 e 44} & \multirow[t]{3}{*}{ Total } & \multirow[t]{3}{*}{$\%$} \\
\hline & \multirow[t]{2}{*}{ Não preenchido } & \multirow[t]{2}{*}{$\%$} & \multicolumn{4}{|c|}{ Preenchido } & & \\
\hline & & & Corretamente & $\%$ & Incorretamente & $\%$ & & \\
\hline 2003 & 10 & 11,0 & 51 & 56,0 & 30 & 33,0 & 91 & 100,0 \\
\hline 2004 & 13 & 11,7 & 64 & 57,7 & 34 & 30,6 & 111 & 100,0 \\
\hline 2005 & 10 & 9,6 & 64 & 61,5 & 30 & 28,8 & 104 & 100,0 \\
\hline Total & 33 & 10,8 & 179 & 58,5 & 94 & 30,7 & 306 & 100,0 \\
\hline
\end{tabular}

O preenchimento incorreto das Declarações de Óbito implica problemas na análise final da causa mortis e, conseqüentemente, na definição de estratégias para redução das mortes maternas.

As causas obstétricas diretas foram as mais subnotificadas, como demonstrado neste estudo, sobretudo as mortes por hipertensão na gestação, as hemorragias, as embolias obstétricas e a infecção puerperal. Há necessidade de estratégias específicas para a redução das mortes por tais complicações, evitáveis em sua maioria.

Verificaram-se, ainda, neste estudo, erros no preenchimento dos campos 43 e 44 da declaração de óbito, reforçando a necessidade de se investir em treinamentos mais adequados para o correto preenchimento das Declarações de Óbito, para que tais campos sejam validados a fim de se classificar o óbito como morte materna declarada.

Laurenti et al. 5 avaliaram uma possível melhora do preenchimento desses campos nas capitais, mas ressaltam que o simples preenchimento, sem menção a uma causa básica materna nas partes I e/ou II da Declaração de Óbito, não remete automaticamente à ocorrência de morte materna, sendo a informação apenas um indicativo de possível morte materna que precisa ser sempre elucidada por meio de investigação.

Sugere-se, portanto, que dois levantamentos sejam feitos, o número de óbitos maternos declarados por extenso no atestado médico do óbito (partes I e II) e o número de óbitos em que foram preenchidos os campos específicos, considerando estes últimos como mortes presumíveis e não mortes maternas declaradas.

Sousa et al. 8 sugerem que o correto preenchimento da declaração de óbito materno, pode ser influenciado pela assistência médica prestada no momento do óbito, pelo tipo de serviço em que se encontrava a mulher no momento do óbito (emergência, UTI, obstetrícia, clínica médica), pelo profissional que prestou assistência e pelo profissional que preencheu a Declaração de Óbito. Destacam os autores que, para utilizar a RMM oficial como indicador válido, especialmente para avaliação do alcance de metas preconizadas de redução da mortalidade materna, é necessário conscientizar os profissionais responsáveis pelo preenchimento da Declaração de Óbito a buscarem informações detalhadas e a investigarem melhor as condições em que ocorreram óbitos das mulheres em idade reprodutiva antes de definir a causa da morte.

Alves 15 investigou 1.258 mortes de mulheres em idade fértil, de algumas regiões de Pernambuco em 2003, identificando 54 mortes maternas, estimando uma razão de mortalidade materna de 77 por 100 mil nascidos vivos. O nível de subnotificação encontrado pela autora foi em torno de $46 \%$ dos óbitos maternos e o fator de ajuste estimado foi 1,9. Comparou estes resultados aos encontrados em estudo semelhante realizado em 1994 e concluiu que a mortalidade materna diminuiu no prazo de 10 anos em Pernambuco, porém o nível de subnotificação das mortes maternas permaneceu o mesmo, corroborando os achados da presente pesquisa no Paraná 15.

\section{Conclusões}

Pode-se afirmar que vem ocorrendo redução da mortalidade materna no Paraná, haja vista que os dados são corrigidos e a RMM está em queda; a subnotificação da mortalidade materna, entretanto, permanece inalterada, como ocorre também em outras localidades e países.

Conclui-se que a subnotificação da mortalidade materna é um problema antigo recorrente e de difícil solução em razão das suas características e peculiaridades.

O fator de ajuste/correção da mortalidade materna no Paraná é semelhante ao encontrado em países desenvolvidos. 
A manutenção da subnotificação das causas maternas pode estar ligada a diferentes fatores, já relatados anteriormente por Sousa et al. ${ }^{8}$ e Volochco 7 , dentre eles o desconhecimento das regras de preenchimento da Declaração de Óbito e de sua importância nas estatísticas vitais, fatores que podem ser amenizados com treinamentos mais incisivos e abrangentes. Todavia outros fatores como o ocultamento voluntário da causa materna por temor de associação à negligência, imperícia ou ato ilegal, são de difícil intervenção, pois dependem da decisão e consciência de cada profissional.

Cabe esclarecer aos profissionais da área, por outro lado, que conforme determinação do Ministério da Saúde, os óbitos maternos serão identificados pelos Comitês de Prevenção da Mortalidade Materna e pela vigilância epidemiológica dos municípios.

Como referem Sousa et al. 8, é necessário conscientizar os profissionais responsáveis pelo preenchimento da Declaração de Óbito de mulheres em idade fértil a buscarem informações detalhadas, investigando melhor as condições em que ocorreu cada óbito, confirmando ou não o óbito materno.

As instituições de ensino médico e os conselhos de medicina poderiam contribuir para melhorar esses índices pela realização de treinamentos sobre o correto preenchimento das De-

\section{Resumo}

Este artigo objetiva analisar a subnotificação dos óbitos maternos no Paraná, Brasil, no período de 1991 2005, detalhando as causas básicas que ocultavam os óbitos maternos e o preenchimento dos campos $43 e$ 44 da Declaração de Óbito em período recente (20032005). Os dados foram obtidos no banco de dados dos Comitês de Prevenção da Mortalidade Materna do Paraná, que investigam os óbitos maternos utilizando o método Reproductive Age Mortality Survey (RAMOS). Evidenciou-se pelos dados estudados que a mortalidade materna reduziu 26\% em 15 anos, no entanto o nível de subnotificação não se alterou, gerando fatores de ajuste que variaram entre 1,6 a 1,8. Conclui-se que é necessário que as escolas médicas e o conselho de medicina orientem de forma mais incisiva e abrangente o correto preenchimento da declaração de óbito, para minimizar esta subnotificação. Os Comitês de Prevenção da Mortalidade Materna no Paraná contribuem para a correção da subnotificação dos óbitos maternos, identificando as reais causas e os determinantes destas mortes, definindo estratégias para sua redução. Ações mais efetivas para redução das mortes maternas evitáveis no estado, ainda são imprescindiveis.

Mortalidade Materna; Sub-Registro; Declaração de Óbito claração de Óbito, e em parceria com os serviços e gestores de saúde buscar soluções concretas para a redução das mortes maternas, que em sua grande maioria é evitável e injusta.

Destaque-se que os Comitês de Prevenção da Mortalidade Materna do Paraná não possuem recursos financeiros próprios para seu funcionamento e para produzir material educativo ou organizar treinamentos sobre ao preenchimento da Declaração de Óbito ou outros temas, embora tenham estas atribuições estabelecidas pelo Ministério da Saúde. No Paraná, com apoio de alguns gestores estaduais, os comitês têm produzido os relatórios e os estudos de caso de óbito materno, além de alguns materiais educativos que têm servido de subsídios para os treinamentos nos serviços e universidades.

Os comitês têm contribuído efetivamente para a vigilância dos óbitos maternos, identificando e corrigindo a subnotificação das mortes maternas, apontando os determinantes e medidas de prevenção da mortalidade materna no Paraná.

O incremento dessas ações por parte dos gestores, com base nas análises e recomendações dos comitês, seria recomendável para uma queda mais acentuada nas taxas da mortalidade materna, configurando assim uma demonstração de respeito à mulher e por extensão aos direitos humanos.

\section{Colaboradores}

Todos os autores contribuíram no levantamento bibliográfico, na análise dos dados e na elaboração do artigo. 


\section{Referências}

1. Organização Pan-Americana da Saúde/Organização Mundial da Saúde. Estratégia regional para a redução da mortalidade e morbidade materna. 26a Conferência Sanitária Pan-Americana, 54ạ Sessão do Comitê Regional. Washington DC: Organização Pan-Americana da Saúde; 2003.

2. World Health Organization. Beyond the numbers: reviewing maternal deaths and complications to make pregnancy safer, 2004. http://www.who.int (acessado em 23/Jun/2007).

3. World Health Organization. Maternal mortality in 2000: estimates developed by 2004. http://www. who.int (acessado em 29/Jun/2007).

4. Organização Mundial da Saúde. Classificação estatística internacional de doenças e problemas relacionados à saúde - décima revisão. São Paulo: Centro Colaborador da OMS para a Classificação de Doenças em Português; 1993.

5. Laurenti R, Mello-Jorge MHP, Gotlieb SLD. A mortalidade materna nas capitais brasileiras: algumas características e estimativas de um fator de ajuste. Rev Bras Epidemiol 2004; 7:449-60.

6. Siqueira AAFS, Rio EMB, Tanaka ACd'A, Schor N, Alvarenga AT, Almeida CA. Mortalidade feminina na Região Sul do Município de São Paulo: qualidade de certificação médica dos óbitos. Rev Saúde Pública 1999; 33:499-504.

7. Volochko A. A mensuração da mortalidade materna no Brasil. In: Berquó E, organizadora. Sexo e vida: panorama da saúde reprodutiva. Campinas: Editora da Unicamp; 2003. p. 229-56.
8. Sousa MH, Cecatti JG, Hardy EE, Serruya SJ. Morte materna declarada e o relacionamento de sistemas de informação em saúde. Rev Saúde Pública 2007; 41:181-9.

9. Ministério da Saúde. Manual dos comitês de mortalidade materna. 2a Ed. Brasília: Ministério da Saúde; 2001.

10. Tanaka ACd'A. Dossiê mortalidade materna. http://www.redesaude.org.br (acessado em 17/ Ago/2007).

11. Ministério da Saúde. IDB 2006. Indicadores e dados básicos. http://www.datasus.gov.br (acessado em 17/Ago/2007).

12. Soares VMN, Martins AL. Trajetória e experiência dos Comitês de Prevenção da Mortalidade Materna do Paraná. Rev Bras Saúde Matern Infant 2006; 6:453-60.

13. Programa das Nações Unidas para o Desenvolvimento. Projeto do milênio das Nações Unidas 2005. Investindo no desenvolvimento: um plano prático para atingir os objetivos de desenvolvimento do Milênio. Visão geral. Nova York: Organização das Nações Unidas; 2005.

14. Ministério da Saúde. Pacto nacional pela redução da mortalidade materna e neonatal. Brasília: Ministério da Saúde; 2004. (Informe da Atenção Básica, Ano V).

15. Alves SV. Maternal mortality in Pernambuco, Brazil: what has changed in ten years? Reprod Health Matters 2007; 15:134-44.

Recebido em 21/Nov/2007

Versão final reapresentada em 24/Mar/2008

Recebido em 28/Mar/2008 